\title{
Upper Arm Contouring with Brachioplasty after Massive Weight Loss
}

\author{
Hyun Ho Han, Min Cheol Lee, Sang Hwa Kim, Jung Ho Lee, Sang Tae Ahn, Jong Won Rhie \\ Department of Plastic and Reconstructive Surgery, The Catholic University of Korea College of Medicine, Seoul, Korea
}

Background As the obese population increases in Korea, the number of patients who are trying to lose weight has been increasing steadily. In these patients, skin laxity and deformation of the body contour occurs, which could possibly be corrected by various body contouring surgeries. Here, we introduce the brachioplasty method and our experience of various body contouring surgeries performed in our center.

Methods From November 2009 to August 2011, five cases of brachioplasty were performed. When the patient presented with sagging of the lateral inframammary crease and bat wing deformity in the axilla, extended brachioplasty was performed; in this case, the deformation of the axilla and lateral chest was corrected at the same time. A traditional brachioplasty was performed when contouring was needed only for skin laxity in the upper arm.

Results Complications, such as hematomas or nerve injuries, were not evident. Some patients experienced partial wound dehiscence due to tension or hypertrophic scars found during the follow-up. In general, all of the patients were satisfied with the improvement in their upper arm contour.

Conclusions Given the demands for body contouring surgery, the number of brachioplasty surgical procedures is expected to increase significantly, with abdominoplasty comprising a large portion of these surgeries. For the brachioplasty procedure, preparation and preoperative consultation regarding design of the surgery by experienced surgeons was important to prevent complications such as nerve damage or hematoma formation.

Keywords Surgery, plastic / Upper extremity / Weight loss
Correspondence: Jong Won Rhie Department of Plastic and Reconstructive Surgery, The Catholic University of Korea College of Medicine, 222 Banpo-daero, Seocho-gu, Seoul 137-701, Korea Tel: +82-2-2258-6142

Fax: +82-2-594-7230

E-mail: rhie@catholic.ac.kr

This work was supported by a Seoul R\&BD Program funded by the Seoul government of Korea (NO. SS110011C0211601) and a grant from Catholic Institute of Cell Therapy in 2013.

No potential conflict of interest relevant to this article was reported.

Received: 2 Jul 2013 • Revised: 8 Aug 2013 • Accepted: 13 Aug 2013

pISSN: 2234-6163 • elSSN: 2234-6171 • http://dx.doi.org/10.5999/aps.2014.41.3.271 • Arch Plast Surg 2014;41:271-276

\section{INTRODUCTION}

As the population of obese people continues to increase in Korea, an increasing number of patients are attempting to lose weight by exercise therapy, medicine, gastric band surgery, or gastrectomy. As a consequence, patients undergo massive weight loss, which may lead to skin laxity and deformation of the body contour. The surgical procedure to correct these prob- lems is called "body contouring surgery." Our previous studies on this subject have introduced this in the form of belt dermolipectomy [1] and lower body-contouring with a thigh lift [2]. Here, brachioplasty is presented as an extension of the aforementioned surgeries. In the United States, 15,457 brachioplasty surgeries were performed during 2012, a rapid increase compared with the 338 surgeries in 2000 [3]. Most of the patients underwent brachioplasty and other body contouring surgeries, 
such as abdominoplasty, thigh lift, breast reduction with mastopexy, and belt dermolipectomy, at the same time or serially as needed. Because there could be skin hooding of lateral parts in the inverted V-shape in patients who had massive weight loss (Fig. 1), it is important to surgically correct the laxity of lateral parts by brachioplasty for good cosmetic results. If the patient had serious lateral laxity, with bat wing deformity in the axilla and prominent skin sagging of the upper lateral body, then this was corrected at the same time. Here, we introduce methods and the possible errors that can occur during the brachioplasty procedure, based on our experience of performing various body contouring surgeries at our center.

\section{METHODS}

From November 2009 to August 2011, a total of five bra- chioplasties were performed. Depending on the status of the patients, abdominoplasty, thigh lift, and breast reduction with mastopexy were performed at the same time or serially. Table 1 provides details on the cases presented in this paper. When the patient exhibited sagging of the lateral inframammary crease and bat wing deformity in the axilla, then extended brachioplasty was performed; in particular, the surgery simultaneously corrected the deformation of the axilla and the lateral chest. When the issue was solely skin laxity in the upper arms, a traditional brachioplasty was performed.

\section{Preoperative design}

Patients whose weight remained stable for more than six months were selected as subjects for the operation. The most important factor for successful surgical outcomes was preoperative planning of the surgery. After the surgeons conferred with

\section{Fig. 1. A 37-year-old woman's body contour (case 3)}

Inverted $\mathrm{V}$ deformity after massive weight loss. (A) Anterior view. (B)

Posterior view.
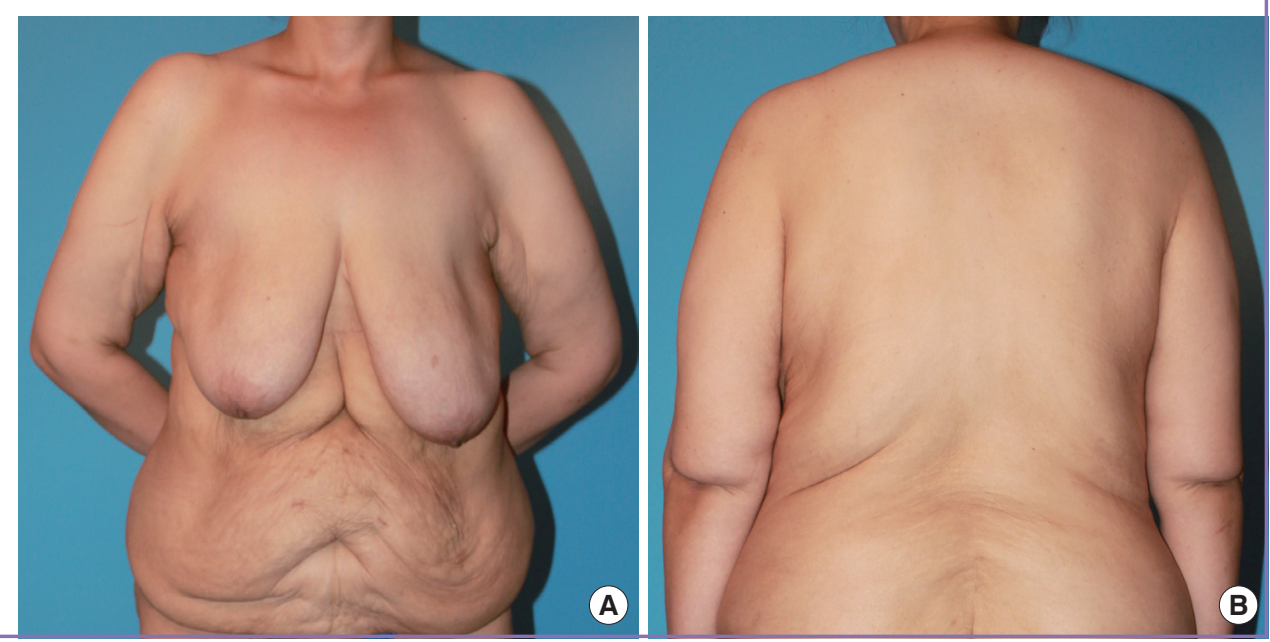

Table 1. Patient summary

\begin{tabular}{|c|c|c|c|c|c|}
\hline Characteristic & Case 1 & Case 2 & Case 3 & Case 4 & Case 5 \\
\hline Sex/Age (yr) & Female/36 & Female/31 & Female/37 & Female/45 & Female/31 \\
\hline Weight-loss method & Gastric bypass & Diet \& exercise & Gastric bypass & Diet \& exercise & Diet \& exercise \\
\hline Body mass index change $\left(\mathrm{kg} / \mathrm{cm}^{2}\right)$ & $48 \rightarrow 24$ & $40 \rightarrow 27$ & $58 \rightarrow 32$ & $38 \rightarrow 31$ & $40 \rightarrow 36$ \\
\hline Pre-/Post- operative weight (kg) & $67 / 65$ & $74 / 70$ & $107 / 100$ & $72 / 71$ & $98 / 96$ \\
\hline Concurrent liposuction at upper arm & No & No & No & Yes & Yes \\
\hline Resected tissue weight (right/left, g) & $140 / 160$ & $200 / 210$ & $342 / 436$ & $86 / 76$ & $1,200 / 800$ \\
\hline Preoperative circumferences & $\begin{array}{c}24-28-31.5-34 \\
27-29.5-32.5-37\end{array}$ & $\begin{array}{c}27-29-35-40.5 \\
27-29-33-39\end{array}$ & $\begin{array}{l}32-35-46-50 \\
32-34-46-49\end{array}$ & $\begin{array}{l}32-36-43-44 \\
32-36-42-43\end{array}$ & $\begin{array}{l}30-33-50-62 \\
27-29-37-44\end{array}$ \\
\hline Postoperative circumferences & $\begin{array}{l}24-27-28-31 \\
27-29-28-33\end{array}$ & $\begin{array}{l}27-27-32-36.5 \\
26.5-27-30-35\end{array}$ & $\begin{array}{l}32-34-38-44 \\
32-33-37-42\end{array}$ & $\begin{array}{l}32-35-38-42 \\
32-35-38-41\end{array}$ & $\begin{array}{l}30-32-46-55 \\
27-29-35-41\end{array}$ \\
\hline Overall surgical procedures & $\begin{array}{l}\text { 1. Abdominoplasty } \\
\text { 2. Brachioplasty } \\
\text { 3. Mastopexy with } \\
\quad \text { augmentation } \\
\text { mammaplasty }\end{array}$ & $\begin{array}{l}\text { 1. Abdominoplasty \& } \\
\text { brachioplasty } \\
\text { 2. Spiral \& vertical medial } \\
\text { thigh lift \& axillary } \\
\text { bulging correction }\end{array}$ & $\begin{array}{l}\text { 1. Belt dermolipectomy } \\
\text { 2. Mastopexy with reduction } \\
\text { mammaplasty \& } \\
\text { brachioplasty } \\
\text { 3. Medial vertical thigh lift }\end{array}$ & $\begin{array}{l}\text { 1. Reduction mammaplasty } \\
\text { \& brachioplasty } \\
\text { 2. Abdominoplasty }\end{array}$ & 1. Brachioplasty \\
\hline
\end{tabular}

"Circumference" denotes the measurement in centimeters of the perimeter of the upper arm divided into four equal parts. The four figures from the left to the right are those of distal to proximal division. The figures in the top row show the measurement of the right arm, and those in the bottom row illustrate the measurement of the left arm. 
the patients, the extent of the procedure was decided 1 day before the operation to allow a thorough prior discussion of the surgery. The patient was first checked for bat wing deformity by holding both arms straight out at a $90^{\circ}$ angle from her body in the standing position. This was followed by marking of the medial epicondyle, axillary crease, and inframammary crease line. In addition to the normal inframammary fold, patients may have additional creases due to skin laxity deformation. Herein, the term "inframammary crease" is used instead of "inframammary fold" in order to avoid confusion. Next, a line connecting the medial epicondyle and the middle point of the axillary crease was drawn, through which the pinch test was performed from the distal to the proximal portion in order to roughly estimate the extent of the area for resectioning. A solid midline of the rough resectioning area was drawn, and then, the incision line was marked with another colored pen (Fig. 2A). A cross-hatch marking was used to facilitate the approximation of the margin after incision and resectioning, which was performed at a later time. During the preoperative planning stage, it was important to keep in mind that if the incision line was extended more distally (i.e., past the medial epicondyle), the medial antebrachial cutaneous nerve innervating the forearm could become injured; scar contracture of the joint in this case may lead to restriction of the range of motion of the elbow.

For patients with bat wing deformity in the axilla, a line was drawn that connected both ends of the axillary crease and the lateral inframammary crease. These patients had skin laxity and ptosis of the breasts in most cases. When these problems were not severe, liposuction was usually sufficient; however, severe cases required breast reduction with mastopexy. In cases in which breast reduction and brachioplasty were performed simultaneously, a line was drawn connecting the extension of the posterior edge of the axillary crease line and the lateral inframammary crease; additionally, a line was drawn that connected the extension of the anterior edge of the axillary crease and the upper line of the breast mastopexy design. The extent of the area for resectioning of the lateral chest tissue was determined by a pinch test. We were careful to ensure that the designed line did not pass the lateral inframammary crease because this might cause lateralization of the breasts and lateral slanting of the newly formed inframammary fold after surgery. After completing the surgical design, the upper arm was divided into four equal parts from the elbow to the axilla, and the circumference of each was measured. A clinical photograph was then taken. Fig. 3 shows the illustrations of the preoperative designs for traditional and extended brachioplasty.

\section{Surgical technique}

The patient was laid in the supine position, and her arms were positioned at a $90^{\circ}$ angle. The distance between the patient's head and the ventilator allowed sufficient room for the procedure. If required, liposuction was performed during the operation, followed by brachioplasty. The surgeons first incised the anterior line of the designed ellipse and then continued to dissect into the upper layer of the muscle fascia. It is important to avoid damage to the muscle fascia because major vessels and nerves are present in the deeper fascial layers. As the dissection approached the axilla, the surgeon dissected saving the tissue of the deep fat layer as much as possible to prevent injury to the lymphatic vessels, intercostobrachial nerve, and medial cutaneous nerve. After passing the designed midline, the range of dissection was widened, taking care not to exceed the optimal range. The optimal

Fig. 2. Example of preoperative brachioplasty design (extended form)

The extent of tissue to excise was determined by pinching the patient in a standing position. (A) Marking by the pinching method is seen here. (B) The closed wound is shown (postoperative day 7).
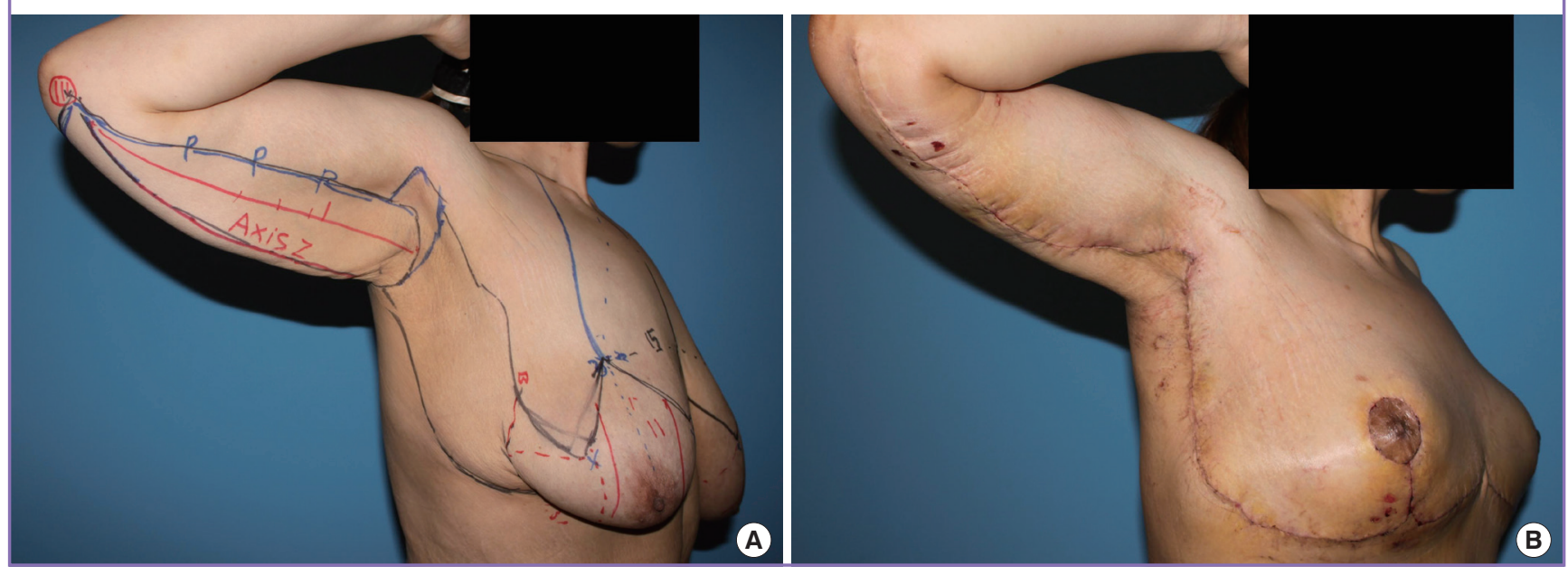


\section{Fig. 3. Illustrations of our preoperative design}

(A) Traditional brachioplasty. (B) Extended brachioplasty.

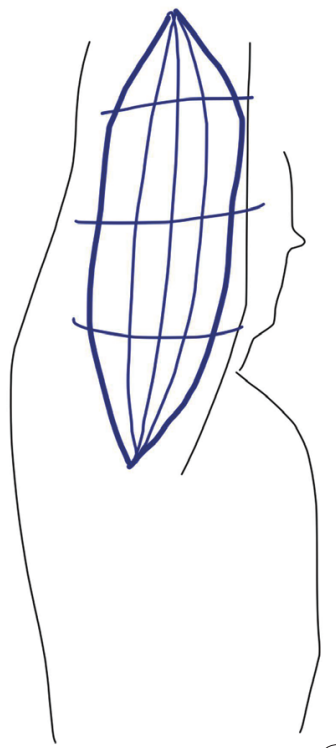

(A)

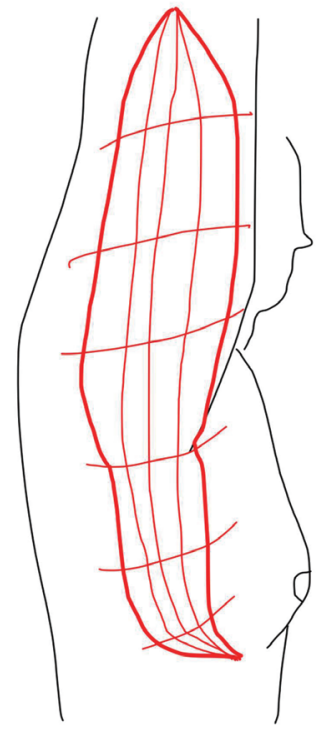

(B) range was determined by taking into account the tension that would be exerted when closing the wound. After checking the ability to close the wound without excessive tension, the posterior line of the designed ellipse was incised in quarters. In this step, the wound was temporarily fixed with metal skin staples to allow the estimation of the tension upon closure.

In extended brachioplasty, the incision was extended to the lateral chest wall and breast. The surgeons first incised the anterior line of the design and then proceeded with the dissection while checking the wound closure. The dissection included the upper layer of the muscle fascia. Because in most cases the inframammary creases were not appropriately located in the lateral portion, the surgeon made a new inframammary fold at a suitable site by attachment of the skin flap to the muscle fascia using a Vicryl 3-0 suture. As mentioned in our previous reports, unlike other surgeons, we did not perform undermining; this minimized the likelihood of hematoma formation and allowed for faster recovery times $[1,2]$. Closed-suction drains were inserted in the resected area, and the deep fascia and the subcutaneous

\section{Fig. 4. Preoperative and postoperative clinical photographs}

Patients satisfied with their improved upper arm contour. (A) Case 2 (preoperative). (B) Case 2 (postoperative month 1). (C) Case 3 (preoperative). (D) Case 3 (postoperative 3 months).
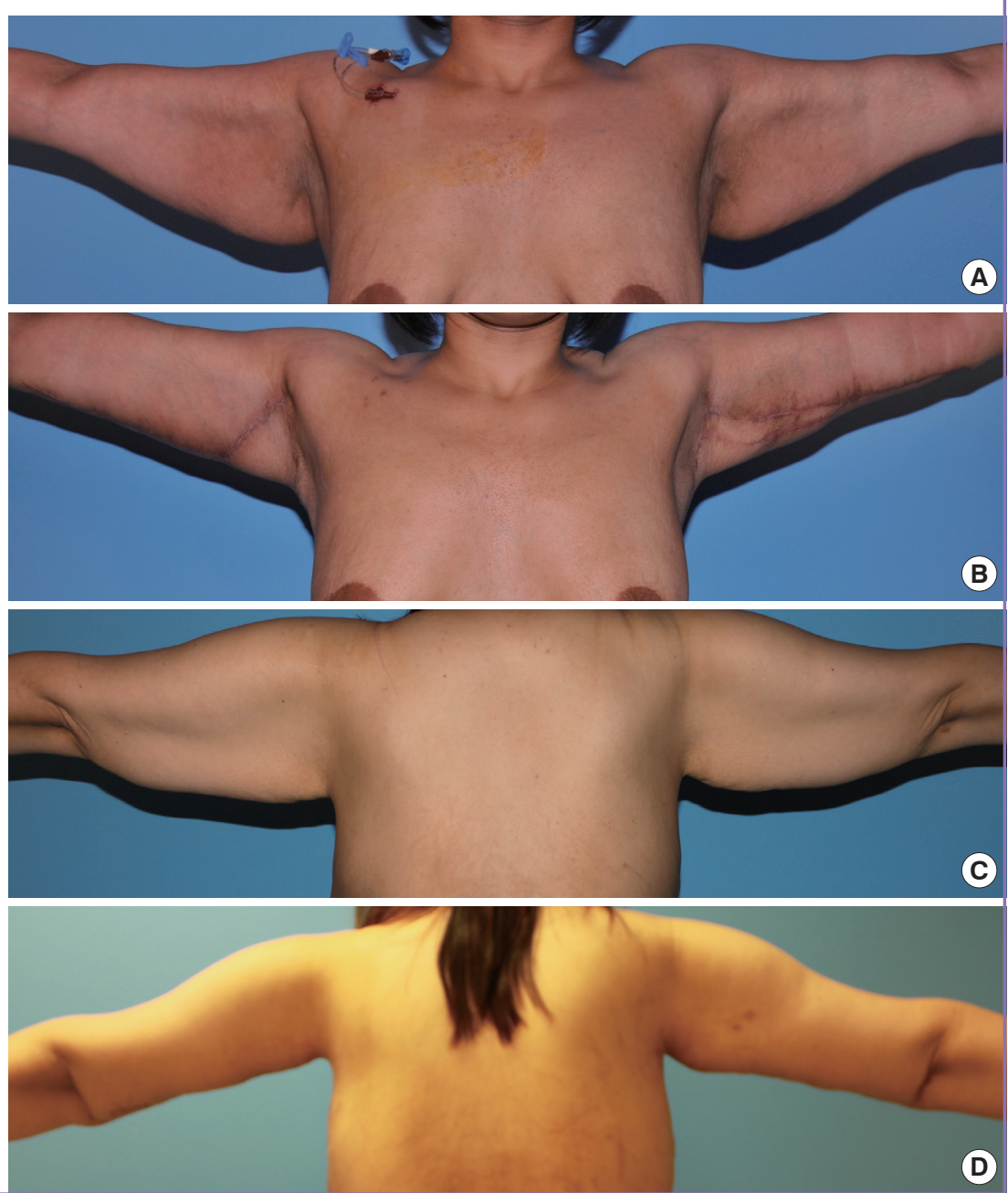
layer were closed using Vicryl 3-0 sutures. When there was no evidence of problems with the skin approximation, the skin was closed with adhesive strips (Fig. 2B). However, when the approximation was not clear, the skin layer was closed with nylon 4-0 sutures.

\section{RESULTS}

In all cases, the drain was removed less than 7 days after the operation, and there were no complications, such as hematoma formation or nerve injury. There were some cases of partial wound dehiscence due to tension; however, this was resolved by secondary healing. Most of the patients presented hypertrophic scars during the follow-up, but in general, all of the patients were satisfied with the dramatic improvement in their upper arm contour (Fig. 4). One patient underwent extended brachioplasty; however, axillary bulging remained after the operation. In this case, the bulging was corrected during a subsequent thigh lift surgery, and the patient was satisfied with the outcome.

\section{DISCUSSION}

In the United States, 15,457 brachioplasty surgeries were performed during 2012, compared with only 338 surgeries during 2000 [3]. However, unlike that in the United States, the obesity rate in Korea has begun to increase only recently; thus, the number of body contouring surgeries has thus far been low. As the obese population in Korea increases, due to the westernization of Koreans' diet, the number of such body contouring surgeries performed is expected to increase. This is also in line with the increase in other plastic surgery procedures, such as those for the eyes or nose. In this situation, it is important to accumulate knowledge and experience of body contouring surgeries such as brachioplasty.

Similar to other body contouring surgeries, the brachioplasty procedure must be carefully designed beforehand. First of all, it is important to precisely set the midpoint of the medial epicondyle of the elbow and the axillary crease. If the midpoint is set appropriately, it is possible to minimize the scars seen from the anterior or posterior direction when the patient is standing still. Further, the surgeon should decide the exact extent of the area to be resected (with breast reduction or to the lateral chest wall) depending on the patient's condition and preferences. Counseling on the expected changes in the body contour following surgery is critical during the preoperative physical examination (e.g., whether or not a patient with bat wing deformity should proceed with the surgery). The extent of surgery has a marked effect on its design. If the surgery is to be extended to the lateral chest and breasts, then it is referred to as "extended brachioplasty." In this case, the procedure should be designed not to pass the lateral side of the inframammary crease, in order to prevent lateral slanting on the newly formed inframammary fold and/or the lateralization of the breasts.

The duration of the brachioplasty procedure should be appropriate. If the surgery becomes prolonged, then the likelihood of swelling is increased, making it more difficult to close the wound. In this case, if closing the wound is forced, the compartment syndrome might occur. Thus, it is important to prepare for the surgery 1 day prior and simulate the procedure beforehand to shorten the duration of the operation.

It is also important to avoid nerve injury. To this end, it is beneficial to dissect superficially into the muscle fascia. Further, it should be ensured that the incision does not pass the elbow in order to avoid injuring the medial antebrachial cutaneous nerve innervating the forearm. Moreover, dissecting the axilla too deeply may cause injury to the lymphatics, intercostobrachial nerve, and medial cutaneous nerve innervating the upper arm.

Some studies reported an upper body lift following a brachioplasty procedure [4]; however, we did not perform an upper body lift if the patient had undergone a spiral thigh lift or belt dermolipectomy. In a circumferential body with serious skin laxity, we observed that if one body contouring surgery had been performed (i.e., an upper body lift, belt dermolipectomy, or spiral thigh lift), then the other two surgeries were not necessary. Our patients received either a belt dermolipectomy or a spiral thigh lift; thus, there were no cases in which both an upper body lift and brachioplasty were performed during the same procedure.

In a spiral thigh lift or classic medial thigh lift procedure, the lower skin flap is hung by the periosteum or deep fascia in many cases to prevent sagging after the operation. However, brachioplasty or the correction of lateral chest wall laxity holds the skin horizontally, similar to the vertical medial thigh lift; thus, the effect of lifting could be maintained without the skin flap being hung by other lifting tissue.

We did not undermine the skin flap during dissection. This was based on past trial-and-error, as well as our concern that excessive undermining raises the probability of hematomas or seromas, and could prolong hospitalization $[1,2]$. In previous reports, hematomas or infections were common complications after brachioplasty [4-6], with $25 \%$ to $40 \%$ reporting major complications after surgery and $3 \%$ to $25 \%$ requiring a second operation [7-9]. For the cases presented here, although the number is quite small, no major complication occurred; this was attributed to the minimization of the undermining of the skin flap.

Massive weight loss of extremely obese people may cause 
general skin laxity of the body; thus, in most cases, various body contouring surgeries are required. In particular, when performing brachioplasty, a greater degree of completion could be achieved by means of careful design and cautious surgical technique to minimize complications.

\section{REFERENCES}

1. Kim JS, Seo JW, Oh DY, et al. Body contouring of breast and abdomen with belt dermolipectomy after massive weight loss: a case report. J Korean Soc Plast Reconstr Surg 2010; 37:681-6.

2. Kim SW, Han HH, Seo JW, et al. Two cases of lower body contouring with a spiral and vertical medial thigh lift. Arch Plast Surg 2012;39:67-70.

3. American Society of Plastic Surgeons. 2012 Cosmetic plastic surgery statistics [Internet]. Arlington Heights, IL: American Society of Plastic Surgeons; c2012 [cited 2013 May 1]. Available from: http://www.plasticsurgery.org/Documents/
news-resources/statistics/2012-Plastic-Surgery-Statistics/ Cosmetic-Procedure-Trends-2012.pdf.

4. Soliman S, Rotemberg SC, Pace D, et al. Upper body lift. Clin Plast Surg 2008;35:107-14.

5. Aly A, Soliman S, Cram A. Brachioplasty in the massive weight loss patient. Clin Plast Surg 2008;35:141-7.

6. Shermak MA. Body contouring. Plast Reconstr Surg 2012; 129:963e-78e.

7. GusenoffJA, Coon D, Rubin JP. Brachioplasty and concomitant procedures after massive weight loss: a statistical analysis from a prospective registry. Plast Reconstr Surg 2008; 122:595-603.

8. Cannistra C, Valero R, Benelli C, et al. Brachioplasty after massive weight loss: a simple algorithm for surgical plane. Aesthetic Plast Surg 2007;31:6-9.

9. Knoetgen J 3rd, Moran SL. Long-term outcomes and complications associated with brachioplasty: a retrospective review and cadaveric study. Plast Reconstr Surg 2006; 117:2219-23. 